\title{
Euthanasia and Nurses Role in It
}

\author{
Baljit Kaur Gill* \\ Associate Professor, School of Nursing and Health Studies, The Open University of Hong Kong, Hong Kong
}

*Corresponding author: Baljit Kaur Gill, Associate Professor, School of Nursing and Health Studies, The Open University of Hong Kong, Hong Kong.

Received Date: October 26, 2019

Published Date: October 31, 2019

\begin{abstract}
The discussion of euthanasia has been aroused in Hong Kong after a teacher's accident which caused him quadriplegia for 25 years. The teacher requested euthanasia, which was unlawful in Hong Kong. After 14 years, the development of euthanasia remains static. Without strong demand and voices in society to change the position of the existing law on euthanasia, public consultation on a proposal of allowing euthanasia has been delayed and not yet accomplished. In this article, it will discuss the situation and progress of euthanasia in Hong Kong. Ethical principles namely autonomy and beneficence and ethical theory (utilitarianism), Code of Ethics and Religion plays a contributing impact on euthanasia. The role of nurses in euthanasia is also important as nurses spent most of the time with the patient. At present, there is no clear guidance for nurses on euthanasia. Their role can be categorized into three phases namely, pre-euthanasia, intra-euthanasia and post-euthanasia stage. The discussion in this paper provide a more concrete and clear description of nurses' role and responsibilities of nurses not only limited to Hong Kong, it can also be used in other countries.
\end{abstract}

Keywords: Euthanasia; Nurse; Hong Kong

\section{Introduction}

The Medical Council of Hong Kong defines euthanasia as "direct intentional killing of a person as part of the medical care being offered". Meanwhile, euthanasia also means "the practice of intentionally ending a life in order to relieve pain and suffering" [1]. Euthanasia can be further classified into voluntary, involuntary and nonvoluntary, active and passive. Voluntary euthanasia is based on one's will like a patient expressed a wish to die while involuntary euthanasia is performed without one's request. Nonvoluntary euthanasia means the person is unable to give informed consent like comatose patients and infants. Taking deliberate steps like injecting lethal medication to end one's life is an active form of euthanasia while causing death by withholding life-sustaining treatments like chemotherapy for cancer patients is a passive form [1].

The aim of euthanasia is to let patients die with dignity. Euthanasia can relieve unbearable suffering for patients with terminal illness in both physical and psychological aspects [2]. In Netherlands, most euthanasia cases are people with cancer, serious heart or lung problems or neurological diseases such as Amyotrophic Lateral Sclerosis (Number of official cases of euthanasia rise $10 \%$ in the Netherlands, 2017) [3]. The physical sufferings include pain, cachexia or exhaustion, Dysphagia and so on. The psychological sufferings are mostly loss of dignity or hopelessness [4]. The medical treatment is futile that would not improve their quality of life. These patients with irreversible physical deterioration can avoid unnecessary suffering which is the greatest benefit of adopting euthanasia.

The following discussion would be divided into four parts, which are

(i) Ethical issues on euthanasia

(ii) Developmental milestone of euthanasia in Hong Kong and other countries

(iii) Barriers and facilitators in legalizing euthanasia in Hong Kong

(iv) A framework of adopting voluntary active euthanasia is drafted to discuss nurses' roles in euthanasia and provide a guideline when euthanasia is legalized in the future.

\section{Ethical Arguments of Euthanasia}

There is no absolute right or wrong of euthanasia as diverse interpretations are drawn from the basis of various ethical principles. Therefore, the views on euthanasia in different ethical perspectives would be discussed. 


\section{Ethical Dilemma in Practice}

The positive side believes that patients should have the right to determine their medical treatment and their lives, coupled with some healthcare providers hold the view that relieving the terminally ill patients from suffering is kind of care [5]. Conversely, doctors are supposed to save patients' life and prevent them to commit suicide, practicing euthanasia seems to be not caring of the patient [6].

\section{Autonomy}

Autonomy is a concept that patients have self-directed plans on their own medical treatment regardless of other's influence, and this can be applicable to individual's decision to end his or her life [7]. In this perspective, euthanasia will be generally considered as a good action in nursing when the action follows patient's autonomy since healthcare providers should respect patient's autonomic decision [8]. Meanwhile, some people suggest that if there are enough reasons to determine euthanasia is morally wrong, autonomy should not be the only reason enjoying superior status than other moral concerns, otherwise, it will weaken the importance of morality [7].

\section{Beneficence}

Beneficence is regarded as doing good to patients, once the healthcare providers assist terminally ill patients to end the existing suffering through euthanasia with sympathetic attitude, this could be viewed as doing good to patients, fulfilling the requirement of beneficence [9]. However, the opposing side proposes that beneficence cannot be a strong reason to support euthanasia as terminating patient's life is equal to patient abandonment, which is regarded as a medical malpractice [9]. In this perspective, euthanasia cannot be regarded as a good practice.

\section{Utilitarianism}

Act-utilitarianism is defined as an act that people select any actions that will promote overall good regardless of circumstances. In this perspective, the factor to decide whether it is right or wrong to cause a human being to death intentionally is the future happiness of that human being [10]. Therefore, if a patient is not suffering from terminal illness and will feel continuous happiness in the coming future, killing will make that patient no longer happy, which euthanasia is regarded inappropriate. Meanwhile, if the patient is suffering from terminal illness with no chance for complete recovery, his/her future life may have more negative elements and no longer be happy, the reason of euthanasia can be justified since it eliminates future suffering of the patient, promoting their overall good.

\section{Code of Ethics and Religious View}

Saving lives of patients is doctor's responsibility and moral duty, if they act against this principle, it is the violation of medical ethics [9]. In terms of religious, it objects euthanasia on the basis of lives are divine and our souls are owned by God who should be the only one controlling human's lifespan. If a doctor conducts euthanasia for patients, it is viewed as an interruption of God's plan which is not acceptable [11].

\section{Development of Euthanasia in Hong Kong}

In 2004, a quadriplegic patient, Tang Siu Bun, wrote a letter to Legislative Council (LegCo) to ask for euthanasia. It attracted media attention and social discussion. According to a local study on attitude towards euthanasia, the general public was found to agree with active euthanasia and was neutral about passive euthanasia [12]. However, the government and society cannot reach a consensus. The Law-making bodies debated the issue of legalizing euthanasia in the same year, but it was hindered by terminological or conceptual confusion [13]. The discussion mainly concentrated on withdrawing life-sustaining treatment in terminally ill patients. It may due to loose usage of term 'euthanasia' in Chinese community [14]. The word 'euthanasia' may be used to describe the standard sense of euthanasia, withdrawing life-sustaining treatment, the state of dying process, or even palliative care [14]. Terminological confusion would impede the discussion of the topic and the development of euthanasia.

Although Tang Siu Bun's case has brought the attention to euthanasia in Hong Kong, euthanasia is still considered as an illegal act in Hong Kong as The Offences Against the Person Ordinance clearly states that "euthanasia involves a third party's acts of intentional killing, manslaughter, or aiding, abetting, counselling or procuring the suicide of another, or an attempt by another to commit suicide". Meanwhile, healthcare professionals are not encouraged to perform euthanasia as seen in the Code of Professional Conduct for the Guidance of Registered Medical Practitioners and the Code of Professional Conduct of Nurses in Hong Kong noting that a practicing nurse should strive to preserve human life and to try to restore bodily functions.

Moreover, Hospital Authority in Hong Kong affirmed its stand against euthanasia on Guidelines on Life-sustaining Treatment in the Terminally Ill and reiterated that euthanasia is illegal and unethical. Even though 'withdraw or withhold life-sustaining treatment' is literally the definition of passive euthanasia, Hospital Authority clearly clarified that the term 'passive euthanasia' should not be used as it contained the meaning of an intention to kill and prevent misleading.

\section{Development of Euthanasia in Other Countries}

Netherlands, Belgium, Luxembourg and Columbia have legalized euthanasia, although Japan has not legalized euthanasia, the court pointed out requirements for active euthanasia. Hong Kong can make a reference from Japan as she was the only example of Asian countries with cultural similarities. In the following, the development of euthanasia in other countries will be highlighted. There were common conditions of conducting euthanasia in the countries mentioned above. First, the patient must suffer from unbearable physical or mental pain that cannot be alleviated, resulting from a serious and incurable disorder. Second, except Columbia, the patient should make a voluntary, well-considered, 
repeated choice, and his/her choice should not result from external pressure.

The aims of legalization in western countries are to bring the matters into the open discussion, to ensure legal safety for patients and medical practitioners, and to establish uniform criteria in case assessment for euthanasia to achieve maximum care. However, the international code of ethics has disagreed with legalizing euthanasia. For example, the Manual of the World Medical Association states that "Euthanasia is the act of deliberately ending the life of a patient, even at the patient's own request or at the request of close relatives, is unethical". The Medical Association also mentioned that "a physician shall always bear in mind the obligation to respect human life". Despite of disagreement of the International Code of Ethics, there were some countries legalized it. Two principles of approving euthanasia adopted in western countries were inferred. Human dignity is one of the principles. Euthanasia has respected human dignity because it can avoid unnecessary suffering and act according to patient's will. Human right is another principle since it puts patient's autonomy and self-determination into consideration.

In western countries, Netherlands first legalized euthanasia in 2002, followed by Belgium in the same year. In the Netherlands, euthanasia was allowed for people with at least 12 years old. Consent of parents or guardians were required for children between 12 and 16 years old. Special conditions of euthanasia included the followings. First, euthanasia was the only reasonable option to deal with patient. Second, the death had to be operated in a medically appropriate fashion with the presence of doctor. Third, a report to a regional committee was needed after finishing euthanasia. In both countries, nurses' role is to assist in decision-making process of that euthanasia patients and to provide opinion to physicians [15]. Luxembourg legalized euthanasia in 2009 available for all age of majority. Some specific criteria listed in the guideline including patient should be conscious at the time of the request with written consent, approval of a panel of experts and two doctors are needed, and it can revoke the request at any time.

While in Asia, Japan is the only country that have an official prerequisite on euthanasia. It has been discussed openly because of the dispute of Tokai University Hospital case in 1995 16, then, the court has pointed out prerequisites for active euthanasia [16]. It was controversial whether these requirements were appropriate and adequate or not to justify active euthanasia in Japan, but it provided a basis for the subsequent cases [16]. Recently, Japan have formulated guidelines concerning the rule for "death with dignity" and tended to establish a guideline system instead of making active euthanasia lawfully [16].

\section{Barriers and Facilitators in Legalizing Euthanasia in Hong Kong}

Apart from the conceptual confusion of "Euthanasia" and conflicts in ethical issues, Chinese culture is another major obstacle impeding the development of euthanasia. Under the influence of Chinese culture, talking about death is disrespectful and viewed as taboo. Filial piety is being emphasized which viewing death or euthanasia is pessimistic. Bowman and Singer indicated that life and dying is related to family issue instead of individual matter in Chinese culture. Patients may consider and respect family's opinion more than his own will [17].

However, Buddhism, the most common religion in China, views euthanasia as the process to achieve "pure land" in which allowing death without suffering [12]. It emphasizes reincarnation and encourages believers to chase peaceful dying. Thus, Buddhism followers in Hong Kong may be more acceptable to euthanasia. Generally, hope represents a positive attitude while encountering adversity. When increased age and serious irreversible illness intersect, people would perceive the situation as hopelessness. Therefore, it is more preferred to alleviate suffering rather than to prolong life.

\section{Measures to Guard Euthanasia}

Currently, the government would rather focus on the enhancement of palliative care services instead of considering legalization of euthanasia as if optimal palliative care can reduce the sufferings of patients. Palliative care services in Hong Kong are mainly provided by public care system with 300 in-patient beds in 16 hospitals. Only 19 palliative care specialists and approximately 600 nurses provide the services while there are 46,000 deaths every year. Enhancing current palliative services would be a huge expenditure, 55.3 billion dollars of financial provision for Hospital Authority may be inadequate. Providing an option of euthanasia can not only shift the resources to other level care, but also bring benefits to terminally ill patients.

In addition, some anti-euthanasia arguments can be resolved through formulating strict conditions. For instance, people may be afraid of abuse of euthanasia like being euthanasia without consent or being pushed by family members to perform euthanasia. In fact, setting conditions like requiring written consent and voluntary request can settle their concerns.

Comparing to other countries, the development of euthanasia in Hong Kong is relatively slow. Therefore, this report goes forward discussing what it would be if legalizing euthanasia and what nurses' roles would be in euthanasia process. A framework is created below to allow a peek for public or stakeholders to see how euthanasia can be run in a nurses' perspective. Conditions of euthanasia-legalized countries and Netherlands' euthanasia protocol were referred to formulate the framework.

To avoid terminological confusion, in this framework, euthanasia is defined as a practice of intentionally ending a life by injecting lethal medication to relieve physical or psychological sufferings based on one's voluntary request.

\section{Suggested Conditions}

The following criteria should be ensure for implementing legal euthanasia.

1. Individual must be at least 18 years old. 
2. The person should be Hong Kong permanent residents to prevent death tourism.

3. The person must suffer from unbearable physical or psychological suffering, dead-end medical situation or terminal phase of illness.

4. The request for euthanasia must be voluntary, informed, well-considered, free from coercion and external pressure.

5. Individual should be competent to sign a written consent.

6. Mutual approval of a panel of experts including clinical psychologist, social worker, case nurse and two doctors is required.

\section{Nurses' Roles in Euthanasia}

Nurses are not the one conducting euthanasia, but they are involved in the process which begins when the patient requests for euthanasia and ends with providing support to the patient's relatives and healthcare colleagues after the act [18]. It is vital for nurses to know their role during the process. Nurses should be open-minded and non-judgmental to accept the euthanasia request as nurses' professional attitude could discourage patient from euthanasia [18]. Nurses' role in euthanasia can be divided into three phases.

\section{Phase I: Pre-Euthanasia}

This is the initial phase of euthanasia from receiving a euthanasia request to sign a written consent. It will be consisted of assessment, consultation and written consent during this phase.

\section{Assessment}

Nurses should listen to the patient carefully, to assess the underlying reasons for requesting euthanasia and the factors contributing to the decision. Improper requests which originate from relievable suffering should be withdrawn and provide alternative methods for the patient to avoid undignified deaths [18]. Also, patients should be assessed the knowledge regarding his or her medical diagnosis, condition, prognosis and other available alternatives, including palliative care to ensure the decision is well-informed as full disclosure is important in executing the right to self-determination freely [19]. Then, general condition such as physical examination, severity of illness and predictable deteriorated condition of the patients should be assessed to ensure that patient fulfills the criteria of conducting euthanasia. Nurses should be familiar with the assessment skills which would speed up the process and enhance the accuracy. Patient's family is also involved to assess their reaction on the request of euthanasia, allow heartfelt communication between patient and family members, encourage ventilation on their emotion, and use active listening skills to identify their needs. Hence, nurses should be sensitive and maintain professional manner when conducting assessment.

\section{Consultation}

As mentioned in the condition, mutual agreement of a panel of experts is needed to proceed to euthanasia. This committees include clinical psychologists, social workers, nurses and doctors. Nurses becomes an advocator which is a representative of the patient providing information about the patient's condition and their relatives' wishes [20] to allow patient's perspective is considered in the consultation.

\section{Written Consent}

Consent should be signed in a quiet room to ensure a nondisturbing environment. Nurses then explains the consent with non-threatening tone and allow time for questions. It is important for nurses to make ensure the patient and family completely understand the process of euthanasia, potential discomfort during the administration and patient right before the signature of written consent. Referring to Belgium, the act of euthanasia would be effect after one month of patient's written request to allow deep contemplation. They can revoke their request within that onemonth period, in which the documents will be removed from the medical record and destroyed.

\section{Phase II: Intra-Euthanasia}

This is the implementation phase of euthanasia from preparing the patient and medication to administering euthanasia. It should be conducted in a special room to provide peaceful environment for promoting human right of die with dignity and minimizing disturbance of the procedures.

\section{Preparation}

Firstly, intravenous access is set on the patient for injecting medication. Secondly, procedure would be explained again to the patient and family members and reassure that nurses will present in the process and give support to relieve their anxiety. Thirdly, nurses assist in preparing medication including sedative agent, analgesic agent and euthanatics and label them properly. An intravenous premedication like midazolam can be administered first to induce a light sleep if the patient does not wish to be aware of the moment of coma induction.

\section{Assistance}

Emergency set should be prepared by nurses and used only under the instruction in protocol. Also, Nurses should offer emotional support to family members if they present in the procedures.

\section{Record}

To evaluate the procedure, nurses should keep a record of all used medication, and involved situations and person. The forms including signed consent forms, pain assessment form, record of euthanasia and last office chart should be charted clearly. The record of euthanasia is similar to the record of operation theatre which needed to record the date and time, place of the procedure, healthcare professionals involved and ranks, the intravenous injection site and all given medication.

\section{Phase III: Post-Euthanasia}

This is the last phase of euthanasia from certifying death of patient to supporting patient's family. After the doctors certified the 
death, nurses can explain to family about the cease of euthanasia and proceed to the last office procedure. Even though the decision is deliberately made, grieving process and feelings of guilt in family members may rise. Emotional support like giving reassurance and listening to their feelings should be provided to them [18]. It requires good communication skills and counselling skills in nurses. Timely referral to counsellor is beneficial for family whose emotion is uncontrolled. In addition, all unused euthanatic agents should return to pharmacy for appropriate disposal and prevention of improper use of euthanatic agents. Incident evaluation form should be filled in for occurrence of unexpected problem like underdose.

\section{Conclusion}

Euthanasia being intensely debated for more two decades globally, was a sensitive topic related to one's own choice and death. It is a highly complicated issue involving implications in different aspects, such as medical, moral, ethical, social and so on. The report reviewed voluntary active euthanasia which refers to an intentional action administered by physicians through injecting lethal drugs to end a life, to relieve the unbearable suffering of the patient. Other classifications of euthanasia were also introduced.

When it comes to determining whether euthanasia should be legalized in a country or not, ethical dilemma occurs. Doctors are expected to save life instead of assisting in suicide, while they must respect patients' right to choose. Four ethical principles and two arguments on euthanasia, including autonomy, beneficence, utilitarianism, right to do wrong, code of ethics and religious view, were deliberated in this report. Although the experience of Tang Siu Bun has caught the public's attention and began to discuss euthanasia more openly in the society, the development of euthanasia in Hong Kong remains stagnant. The government did not plan to legalize euthanasia in Hong Kong, alternatively, the end-of-life care was mainly focused on palliative care, advance directives ad advance care planning. Meanwhile, Netherlands, Belgium, Luxembourg and Columbia have legalized euthanasia under strict conditions as well as Japan which pointed out four prerequisites for active euthanasia. Comparing with other countries, the development of end-of-life care in Hong Kong still has room for improvement. Barriers and facilitators in legalizing euthanasia were explained. The barriers included conceptual confusion, conflicts in ethical issues and Chinese culture, while Buddhism and hope were the facilitators.

Euthanasia not only can shift medical resources to other level of care, but also bring benefits to terminally ill patients. Hence, a framework on how to run euthanasia in nurses' perspective in Hong Kong was built. Seven conditions of performing euthanasia were suggested. There were three phases of carrying out euthanasia, including pre-euthanasia, intra-euthanasia and post-euthanasia. The role of nurses in each phases of euthanasia was emphasized. In the pre-euthanasia phase, nurses must equip with professional assessment skills and communication skills, and act as an advocator to protect patients' rights. During the intra-euthanasia phase, nurses should assist in preparing medication, monitor the patient's condition, document properly and provide support to the family members. In the post-euthanasia phase, nurses can provide emotional support to the relatives, make referral if necessary, return the unused agents to pharmacy and fill in incident evaluation form.

Since euthanasia is a controversial issue, legalization takes time. As a nurse, we have the responsibility to promote the acceptance of euthanasia to provide one more optimal end-oflife choice for patients. Due to the inactive atmosphere, limited surveys and researches on this issue, we can work on conducting more studies and encouraging open discussion in society. This report is a tentative discussion that more professional research and public discussion should be promoted. Further development of euthanasia and modification of the above protocol are needed through exploring diverse evidences and opinions. It is hope that there is a great progress towards euthanasia.

\section{Acknowledgement}

The author, Baljit Kaur Gill, would like to thank Bobby Gill for the support, insight and guidance of writing this article.

\section{Conflict of Interest}

The author declares that there is no conflict of interest. No funding has been received for the conduct of this article.

\section{References}

1. MacKinnon B, Fiala A (2014) Ethics: Theory and contemporary issues: Nelson Education.

2. Van Alphen JE, Donker GA, Marquet RL (2010) Requests for euthanasia in general practice before and after implementation of the Dutch Euthanasia Act. Br J Gen Pract 60(573): 263-267.

3. Onwuteaka Philipsen BD, Van der Heide A, Muller MT (2005) Dutch experience of monitoring euthanasia. Bmj 331(7518): 691-693.

4. Smets T, Bilsen J, Cohen J, Rurup ML, Deliens L (2010) Legal euthanasia in Belgium: characteristics of all reported euthanasia cases. Med Care 48(2):187-192.

5. Landry JT, Foreman T, Kekewich M (2015) Ethical considerations in the regulation of euthanasia and physician-assisted death in Canada. Health policy 119(11):1490-1498.

6. Gillett G, Chamberlain J (2013) The clinician's dilemma: Two dimensions of ethical care. International journal of law and psychiatry 36(5-6):454460.

7. Varelius J (2013) Voluntary euthanasia, physician-assisted suicide, and the right to do wrong. HEC forum 25(3): 229-243.

8. Quaghebeur T, Dierckx de Casterlé B, Gastmans C (2009) Nursing and euthanasia: a review of argument-based ethics literature. Nurs Ethics 16(4): 466-486.

9. Himchak MV (2011) A social justice value approach regarding physicianassisted suicide and euthanasia among the elderly. Journal of Social Work Values and Ethics 8(1): 1-14.

10. Singer P (2003) Voluntary euthanasia: a utilitarian perspective. Bioethics 17(5-6): 526-541.

11. Stylianidou S (2013) Terminal cancer patients and euthanasia the church's position. Hellenic Journal of Surgery. 85(2): 105-108.

12. Ming lin Chong A, Fok Sy (2005) Attitudes toward euthanasia in Hong Kong-A comparison between physicians and the general public. Death Stud 29(1): 29-54.

13. Liu A (2009) Ah Bun and Euthanasia in Hong Kong. Hong Kong LJ 39: 147. 
14. Van TSE C, PANG SMC (2006) Euthanasia and forgoing life-sustaining treatment in the Chinese context. Death, dying and bereavement: A Hong Kong Chinese experience 1: 169.

15. Francke AL, Albers G, Bilsen J, de Veer AJ, Onwuteaka Philipsen BD (2016) Nursing staff and euthanasia in the Netherlands. A nation-wide survey on attitudes and involvement in decision making and the performance of euthanasia. Patient Educ Couns 99(5): 783-789.

16. Kai K (2010) Euthanasia and Death with Dignity in Japanese Law. J Int Bioethique 21(4): 135-147.
17. Bowman KW, Singer PA (2001) Chinese seniors' perspectives on end-oflife decisions. Soc Sci Med 53(4): 455-464.

18. De Casterlé BD, Verpoort C, De Bal N, Gastmans C (2006) Nurses' views on their involvement in euthanasia: a qualitative study in Flanders (Belgium). J Med Ethics 32(4): 187-192.

19. Mathieson S (2013) Live and Let Die: The legalisation of euthanasia in New Zealand.

20. Inghelbrecht E, Bilsen J, Mortier F, Deliens L (2010) The role of nurses in physician-assisted deaths in Belgium. Cmaj 182(9): 905-910. 